\title{
Angle-resolved ballistic phonon absorption spectroscopy in the lowest Landau level
}

\author{
C.J. Mellor * , U. Zeitler ${ }^{1}$, A.M. Devitt, S.H. Roshko, A.J. Kent, K.A. Benedict, \\ T. Cheng, M. Henini \\ Department of Physics, University of Nottingham, Nottingham NG7 2RD, UK
}

\begin{abstract}
We report the results of a study of the absorption of non-equilibrium ballistic phonons by a two-dimensional electron system, in strong magnetic fields, by measuring its change in resistance. At the even denominator Landau level filling factors, $v$, of $1 / 2$ and $3 / 2$ the electron-phonon interaction is observed to be cut-off above a particular value of the component of the wavevector perpendicular to the low dimensional system. This cut-off is consistent with that due to the finite thickness of the 2DES. At fractional quantum Hall effect minima $(1 / 3,2 / 5,2 / 3)$ we observe that the cut-off to the interaction depends on the filling factor. At $v=1 / 3$, the energy gap determined from these experiments is consistent with the theoretical prediction of the magnetoroton energy gap. (c) 1998 Elsevier Science B.V. All rights reserved.
\end{abstract}

Keywords: Fractional quantum Hall effect; Phonon spectroscopy; Magnetoroton

\section{Introduction}

The interaction of phonons with a two-dimensional electron system (2DES) in the fractional quantum Hall $(\mathrm{FQH})$ regime has been a topic of great interest in recent years using experimental techniques such as time-averaged phonon spectroscopy [1], the temperature dependence of the resistance [2], thermopower experiments [3] and phonon-emission experiments [4]. Despite the weak electron-phonon interaction, phonons are able to couple efficiently to a 2DES as conserva-

\footnotetext{
* Corresponding author. Fax: +44 115 9515180; e-mail: chris.mellor@nottingham.ac.uk

${ }^{1}$ Present address: Institut für Festkörperphysik, Abteilung Nanostrukturen, Universität Hannover, Appelstraße 2, D30167, Germany.
}

tion of both in-plane wavevector and energy is possible.

Different mechanisms are expected to dominate the electron-phonon interaction depending on the filling factor, $v$, of the Landau level. At even denominator filling factors such as $v=1 / 2$ and $3 / 2$ it is believed that the 2DES can be viewed as a Fermi liquid of composite fermions with a well-defined Fermi wavevector, $k_{\mathrm{F}}[5]$. Work with $2 \mathrm{DES}$ in zero magnetic field suggests that this can lead to an effective cut-off to the electron-phonon interaction when the in-plane phonon wavevector exceeds $2 k_{\mathrm{F}}$. The finite thickness of the 2DES also limits the phonon momentum perpendicular to the 2DES that can be absorbed. This greatly reduces the electron-phonon interaction in a heterojunction for perpendicular wavevectors greater than $1 / a_{0}$ where $a_{0}$ is a measure of the thickness of the 2DES. 
At an odd denominator filling factor, the 2DES forms an incompressible fractional quantum Hall effect state. The low lying excited states of these quantum liquids are believed to consist of collective modes with a deep minimum in their dispersion relation, known as the magnetoroton minimum, at wavevectors comparable to $l_{B}^{-1}$ where $l_{B}$ is the magnetic length [6]. In recent years these excitations have been investigated experimentally by means of resonant Raman scattering techniques [7] and time-averaged phonon absorption spectroscopy [1]. The resonant Raman scattering experiments rely on disorder present in the sample to couple the photons to the large in-plane wavevectors of the magnetorotons. Phonon techniques have the potential to probe the magnetoroton minimum with a known range of in-plane wavevectors.

The experiments described in this paper are the first experiments in which the direct absorption of ballistic phonons by a 2DES in strong magnetic fields have been observed using a time-resolved experiment with a time resolution much better than the typical phonon time of flight. An earlier, preliminary, report has been published elsewhere [8].

\section{Experimental}

The two samples used in these experiments are heterojunctions grown on a $2 \mathrm{~mm}$ semi-insulating GaAs substrate. The properties of the two samples are summarised in Table 1. Due to the extremely small effect of the phonon absorption on the resistance of the 2DES [8], it has been patterned into a meandering path that covers a $5 \times 5 \mathrm{~mm}^{2}$ area (sample A) or a $1 \times 1 \mathrm{~mm}^{2}$ area (sample B). The length to width ratio of the meander is $\sim 300$ for both samples. In order to avoid the effects of contacts being heated by the phonon pulse the meander is positioned to one end of the $11 \times 11 \mathrm{~mm}^{2}$

Table 1

Carrier density and zero field mobility of the two samples

\begin{tabular}{lll}
\hline & Sheet density $\left(10^{15} \mathrm{~m}^{-2}\right)$ & Mobility $\left(\mathrm{m}^{2} \mathrm{~V}^{-1} \mathrm{~s}^{-1}\right)$ \\
\hline Sample A & 1.16 & 150 \\
Sample B & 1.4 & 140 \\
\hline
\end{tabular}

sample. The contacts are positioned in the two opposite corners of the sample. This allows the experimental readings to be completed before the ballistic phonons reach the contacts. On the rear surface facing the centre of the meander line, a thin film constantan heater, also in the form of a $1 \times 1$ $\mathrm{mm}^{2}$ meander was fabricated. On sample B an additional heater was positioned so that the centre of the heater subtended an angle of $45^{\circ}$ to the centre of the meander line. The majority of phonons incident on the 2DES from this heater propagate in the $\left[\begin{array}{lll}1 & 1 & 0\end{array}\right]$ plane.

The sample was mounted, in vacuo, on the tail of a dilution refrigerator. The meander line was electrically connected to a room temperature preamplifier using a coaxial cable with a total capacitance between room temperature and the sample of $30 \mathrm{pF}$. A pulse of non-equilibrium phonons was created by applying a short voltage pulse, typically $50-100 \mathrm{~ns}$, to the thin film heater. The nonequilibrium temperature, $T_{\mathrm{h}}$, of phonons entering the cold substrate can be calculated from the power, $P_{\mathrm{h}}$, dissipated in the heater using acoustic mismatch theory [9] as $P_{\mathrm{h}}=\sigma A_{\mathrm{h}}\left(T_{\mathrm{h}}^{4}-T_{0}^{4}\right)$. Here $\sigma=524 \mathrm{~W} \mathrm{~m}^{-2} \mathrm{~K}^{-4}, A_{\mathrm{h}}$ is the surface area of the heater and $T_{0}$ the substrate temperature.

At sufficiently low temperatures, the phonons travel ballistically through the GaAs and are incident on the 2DES with an angular resolution determined by geometric considerations and phonon focussing. A small proportion of the incident phonon flux is absorbed by the 2DES. This absorbed power causes a rise in the temperature of the 2DES. In the experiment, the temperature increase is deduced from the change in the 2DES resistance which is calibrated against temperature under equilibrium conditions. As the measurements are made on the time scale of the order of $50 \mathrm{~ns}$, whereas the time scale for internal equilibration in the 2DES is at least an order of magnitude faster, it is reasonable to use the resistance change of the meandering sample to determine an increase in electron temperature.

A DC current (typically $50 \mathrm{nA}$ ) is passed through the 2DES. The phonon response is measured by recording the voltage across the meander as a function of time and averaging $10^{3}-10^{6}$ traces. The repetition rate is limited to $10-100 \mathrm{~Hz}$ to min- 


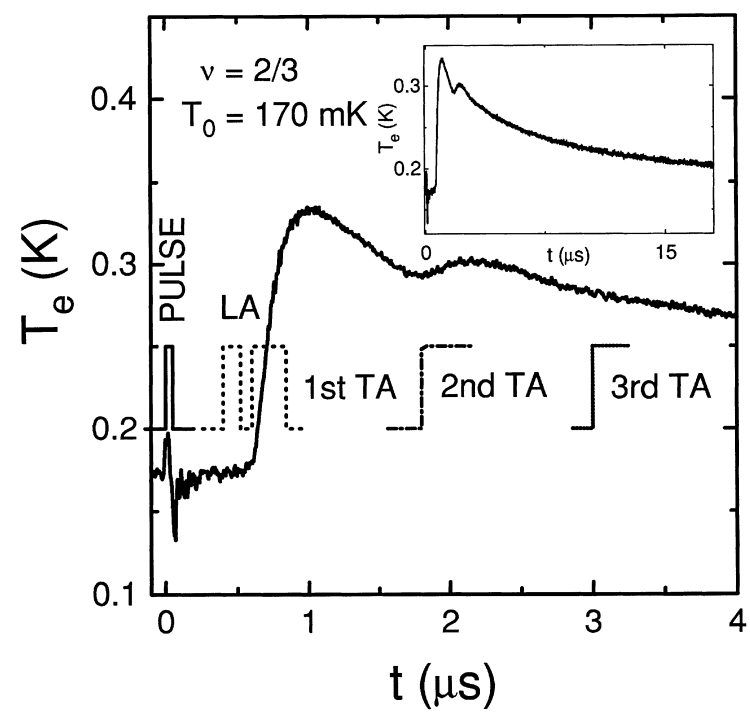

Fig. 1. Phonon response (at $T_{0}=170 \mathrm{mK}$ ) showing the variation of the 2DES temperature of sample B at $v=2 / 3(8.66 \mathrm{~T})$ after a $50 \mathrm{~ns}$ pulse of $1.45 \mathrm{~K}$ phonons has been emitted from the heater directly under the sample at $t=0$. Arrival times of different phonon polarisations are also shown. Inset shows 2DES temperature at longer times.

imise the effects of substrate heating and to allow the substrate to cool down before the next pulse of phonons is applied. The electrical time response of the system is determined by its $R C$ time constant of $30-150 \mathrm{~ns}, C \approx 30 \mathrm{pF}$ is the cable capacitance between sample and amplifier and $R \approx 1-5$ $\mathrm{k} \Omega$ is the (variable) input impedance of the preamplifier in parallel with the two-point resistance of the meander.

In Fig. 1, the typical response of the 2DES (sample B) at a filling factor $v=2 / 3$ to a pulse of non-equilibrium phonons from the heater directly beneath the meander is shown. Initially the 2DES is at a temperature of $170 \mathrm{mK}$. After 0.4 $\mu \mathrm{s}$, LA phonons travelling directly across the wafer have traversed the $2 \mathrm{~mm}$ thick GaAs substrate and hit the 2DES. This leads to only a very small increase in its temperature showing that the interaction with these LA phonons only plays a minor role in the phonon absorption. After $0.6 \mu \mathrm{s}, \tau_{\mathrm{TA}}$, the temperature of the 2DES rises much more rapidly. This coincides with the time that TA phonons travelling almost perpendicular to the interface hit the 2DES. The electronic rise time is fast enough to record the true rate of change of electron temperature with time. The rise time is due to the length of the phonon pulse and the angular spread of the phonons (geometrically the maximum angle is $\sim 27^{\circ}$ ). Subsequently with no hot ballistic phonons present in the vicinity of the 2DES, the system starts to cool. At a time corresponding to $3 \tau_{\mathrm{TA}}=1.8 \mu \mathrm{s}$ and $5 \tau_{\mathrm{TA}}=5 \mu \mathrm{s}$, further heating is observed. This confirms the interpretation of the heating as being due to TA phonons and demonstrates ballistic propagation of the phonons even over such long distances. Only after several microseconds do the hot phonons thermalise via inelastic scattering processes inside the substrate leading to a rise in the substrate temperature, $T_{\mathrm{b}}$. The electrons only reach thermal equilibrium with the substrate after thermal relaxation of the hot 2DES. We can determine $T_{\mathrm{b}}$ experimentally by measuring the temperature of the 2DES at long times (as shown in the inset of Fig. 1). It is in good agreement with the expected behaviour where $T_{\mathrm{b}}$ is calculated from the total energy, $E_{\mathrm{ph}}=$ $\sigma A_{\mathrm{h}}\left(T_{\mathrm{h}}^{4}-T_{0}^{4}\right) \tau$, dissipated in the heater and the total specific heat of the substrate, $C_{\mathrm{s}}$. This confirms the reliability of the thermometry. Finally, the substrate cools to its initial temperature $T_{0}$ on a time constant determined by $C_{\mathrm{s}} / \Lambda \approx 0.5 \mathrm{~ms}$ where $\Lambda$ is the thermal conductance between the sample and its heatsink.

\section{Even-denominator filling factors}

At a filling factor of $v=1 / 2$ (Fig. 2) we observe a ballistic response of the 2DES to the non-equilibrium phonons. In general, for the same pulse of non-equilibrium phonons, the increase in the electron temperature due to the absorption of ballistic phonons is less than at fractional quantum Hall effect filling factors such as $v=1 / 3,2 / 3,2 / 5$.

When the phonon pulse reaches the 2DES, a small proportion of the incident phonon energy, $\mathrm{d} E_{\mathrm{ph}}=r\left(T_{\mathrm{h}}\right) P_{\mathrm{h}} \mathrm{d} t$ is absorbed in a time interval $\mathrm{d} t$. Here, $P_{\mathrm{h}}$, is the power dissipated by the heater in the form of ballistic phonons and $r\left(T_{\mathrm{h}}\right)$ is the fraction of phonons absorbed by the 2DES when the non-equilibrium phonon spectrum has a tem- 


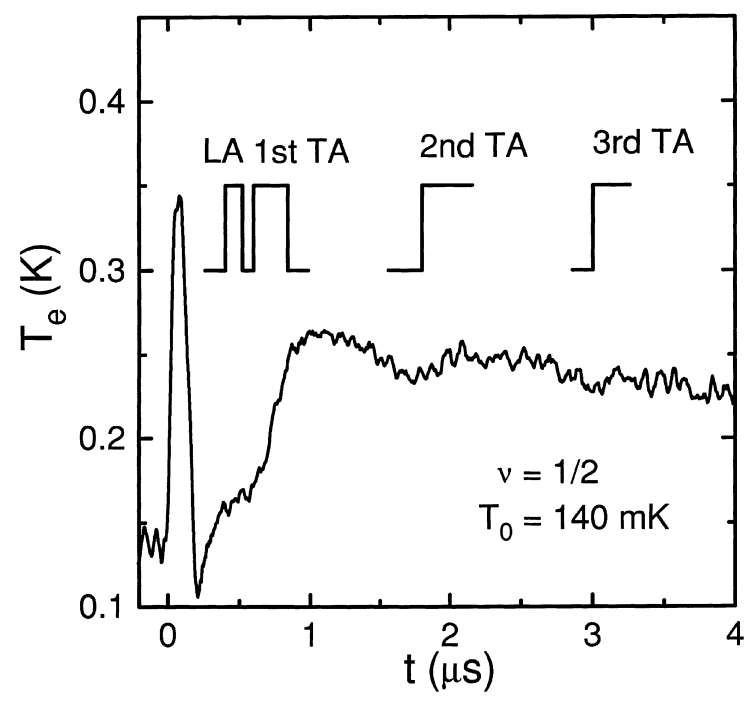

Fig. 2. Phonon response (at $T_{0}=140 \mathrm{mK}$ ) showing the variation of 2DES temperature of sample B at $v=1 / 2(11.54 \mathrm{~T})$ for an identical phonon pulse to that shown in Fig. 1.

perature, $T_{\mathrm{h}}$. Normally, $r\left(T_{\mathrm{h}}\right) \propto\left(T_{\mathrm{h}}^{n}-T_{1}^{n}\right)$ where $T_{1}$ is the lattice temperature [10]. The exponent $n \approx 3$ for piezoelectric coupling and 5 for deformation potential coupling. In the case described here, $T_{\mathrm{h}}$ is much greater than $T_{1}$ and so the fraction of phonons absorbed will depend only on $T_{\mathrm{h}}$. The energy absorbed by the 2DES will manifest itself as an increase in the electron temperature from its equilibrium value $T_{0}$ to a non-equilibrium temperature $T_{1}$ given by,

$\int_{T_{0}}^{T_{1}} \frac{C\left(T_{\mathrm{e}}\right)}{r\left(T_{\mathrm{h}}\right)} \mathrm{d} T_{\mathrm{e}}=P_{\mathrm{ph}} \tau$,

where $C\left(T_{\mathrm{e}}\right)$ is the total specific heat of the 2DES.

At $v=1 / 2$, the heat capacity of the composite fermions is predicted to be linear in temperature (except for a small logarithmic correction) [11]. We therefore approximate the heat capacity as $C=\alpha T$. Inserting this into Eq. (1), we find that,

$r\left(T_{\mathrm{h}}\right)=\alpha\left(T_{1}^{2}-T_{0}^{2}\right) / 2 P_{\mathrm{ph}} \tau$.

If we calculate $r\left(T_{\mathrm{h}}\right) / \alpha$ from our data we find that the fraction of phonons absorbed peaks at a particular value of $T_{\mathrm{h}}$ (Fig. 3). Comparing the results from the direct and angled heaters (sample B) we find that the heater temperature at which $r$ is a

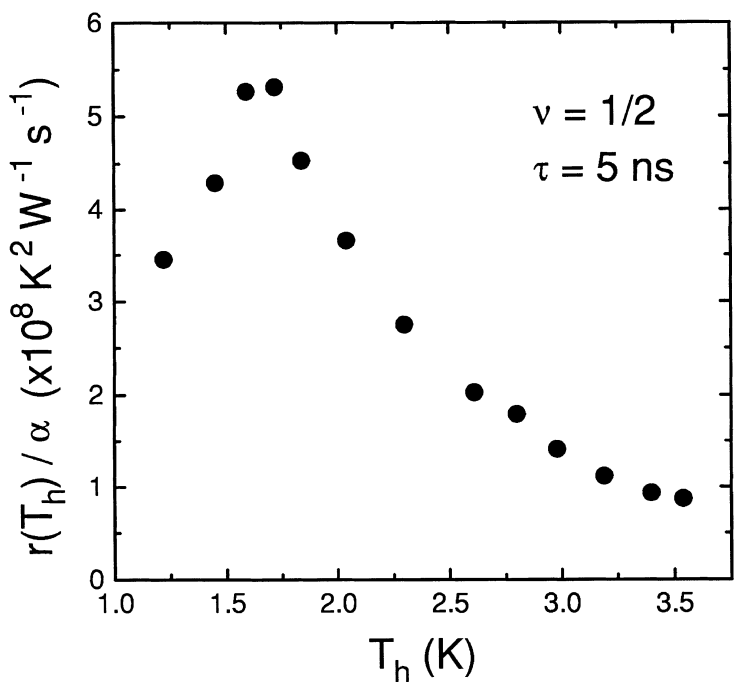

Fig. 3. Variation of $r\left(T_{\mathrm{h}}\right) / \alpha$ as a function of phonon temperature at $v=1 / 2$ (Sample B) for a $5 \mathrm{~ns}$ pulse of TA phonons from heater directly under the 2DES.

maximum differs in a way that is consistent with a $1 / a_{0}$ cut-off. The value of $a_{0}$ is found to be $\sim 5$ $\mathrm{nm}$. The perpendicular component of the phonon wavevector is calculated using the time of flight and considerations of phonon focussing to estimate the incident angles. Similar results are found at $v=3 / 2$.

The $2 k_{\mathrm{F}}$ cut off at $v=1 / 2$ has not been observed in these heterojunction samples as it occurs at a higher wavenumber than the $1 / a_{0}$ cutoff. The decrease in the electron-phonon interaction when the perpendicular component of the wavevector is greater than $\sim 1 / a_{0}$ is much stronger than when the in-plane component of the wavevector exceeds $2 k_{\mathrm{F}}$. At $v=3 / 2$, the predicted $2 k_{\mathrm{F}}$ cutoff would occur at approximately the same phonon temperature as the $1 / a_{0}$ cutoff. However in this case the thermal smearing of the composite fermion Fermi surface $\left(E_{\mathrm{F}} \sim 1 \mathrm{~K}\right)$ may have prevented us from observing it.

At $v=1 / 2$, all phonon energies up to the $1 / a_{0}$ cut-off can be absorbed but also emitted by the 2DES. This leads to a decrease in the relative energy, $\varepsilon$, absorbed as a function of the pulse length, $\tau$. In saturation, the rate of phonons absorbed and emitted by the 2DES are equal and consequently 


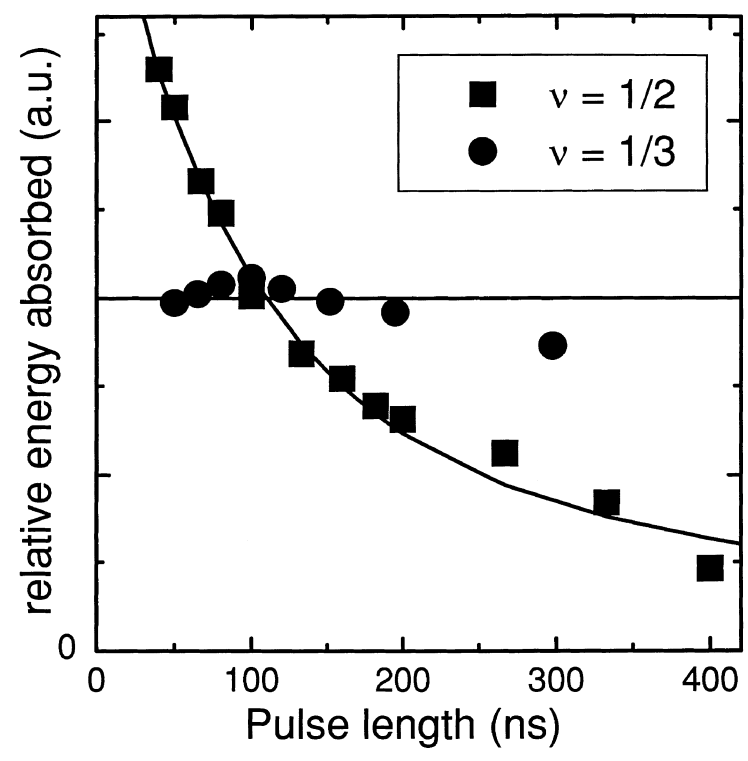

Fig. 4. Total energy absorbed by the 2DES (sample A) at $v=$ $1 / 2$ and $v=1 / 3$ as a function of the length of the phonon pulse. Solid line is a fit of saturation behaviour to the data at $v=1 / 2$ as described in the text.

the 2DES does not absorb any net energy, even if the phonon pulse is present for longer times. In Fig. 4, this behaviour is plotted for Sample A and compared to saturation behaviour $\varepsilon \propto \tau$ $\left(1-\exp \left(-\tau / \tau_{\mathrm{s}}\right)\right)$. Good agreement is found for a saturation time, $\tau_{\mathrm{s}}$, of $55 \mathrm{~ns}$. The value of the saturation time is much longer than is observed in a 2DES in zero magnetic field.

In summary, at $v=1 / 2$ and $3 / 2$ we observe ballistic phonon behaviour similar to that observed in lower mobility 2DES and 2DHS in zero magnetic field. The value of $a_{0}$ is consistent with that expected for a heterojunction of this electron density. The incident angles of the phonons can be estimated from phonon focussing and is confirmed by the self-consistent analysis of the behaviour at $1 / a_{0}$.

\section{Results at $v=1 / 3$}

We report here the results of an experiment on sample $\mathrm{A}$, at $v=1 / 3$, that is time-resolved, but in which the 2DES can absorb phonons over a wide range of angles. It is expected that under these con- ditions the phonon absorption will be dominated by absorption at the magnetoroton minimum. Eq. (1) is still valid in the odd-denominator fractional quantum Hall regime, however the heat capacity $C\left(T_{\mathrm{e}}\right)$ and the fraction of phonons absorbed $r\left(T_{\mathrm{h}}\right)$ will be different due to the presence of the magnetoroton energy gap, $\Delta_{\mathrm{MR}}$. As the excited magnetorotons at $v=1 / 3$ are neutral excitations they cannot contribute directly to an increase in resistance. However, provided they equilibrate with the charged excitations inside the 2DES quickly enough, the energy absorbed will be manifest as an increase in the temperature of the 2DES. To test this hypothesis, we can measure the dependence of the phonon absorption process on the duration of the phonon pulse. The results are shown in Fig. 4. The total relative energy absorbed, defined as the ratio of the absorbed energy to the energy in the non-equilibrium phonon pulse, is constant. In other words, the total energy absorbed by the 2DES is always proportional to the total energy emitted by the heater, with no saturation observed even at times as long as $300 \mathrm{~ns}$. We conclude that the excited magnetorotons do not come into thermal equilibrium with the ballistic phonons by emitting phonons of the same energy and wavevectors on the timescales mentioned above. They must therefore equilibrate by heating the 2DES internally without phonon emission into the substrate.

By measuring the phonon absorption as a function of substrate temperature, $T_{0}$, at a fixed heater temperature and pulse length, we can measure the quantity $C\left(T_{\mathrm{e}}\right) / r\left(T_{\mathrm{h}}\right)$. The simplest guess is that the heat capacity is again linear in temperature. The relative proportion of phonons absorbed, $r\left(T_{\mathrm{h}}\right)$, is expected to be independent of the electron temperature, $T_{\mathrm{e}}$, for $T_{\mathrm{e}} \ll \Delta_{\mathrm{MR}} / k_{\mathrm{B}}$ when the quasi-particle ground state of the 2DES is nearly full and the excited states are almost empty. Therefore, for the temperature range considered, we can regard $r\left(T_{\mathrm{h}}\right)$ as independent of $T_{\mathrm{e}}$ (within an accuracy of $\left.\exp \left(-\Delta_{\mathrm{MR}} / k_{\mathrm{B}} T_{\mathrm{e}}\right)\right)$. The results of such an experiment are shown in Fig. 5. The quantity $C\left(T_{\mathrm{e}}\right) / r T_{\mathrm{e}}$ is approximately constant below 400 $\mathrm{mK}$. Above $400 \mathrm{mK}$ we observe a rapid increase. Assuming that $r$ is constant for a given $T_{\mathrm{h}}$, the best fit to the data suggests that $C\left(T_{\mathrm{e}}\right)=A T_{\mathrm{e}}+B T_{\mathrm{e}}^{7}$. 


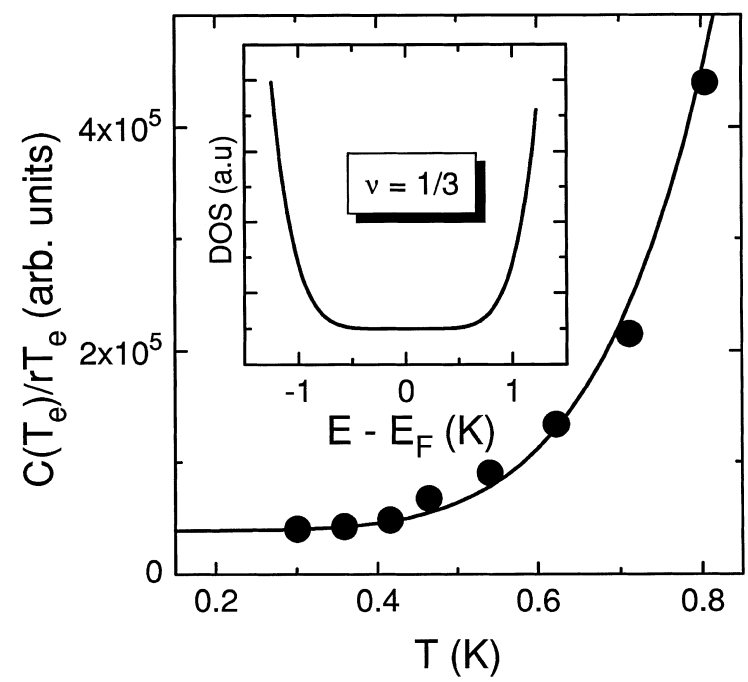

Fig. 5. Variation of $C\left(T_{\mathrm{e}}\right) / r T_{\mathrm{e}}$ as a function of initial 2DES temperature (Sample A) for fixed phonon temperature and pulse length (see text) at $v=1 / 3$. Solid line shows fit that takes into account only the $T_{\mathrm{e}}$ and $T_{\mathrm{e}}^{7}$ terms in the Sommerfeld expansion of the specific heat. The inset sketches the composite fermion density of states at $v=1 / 3$ as extracted from the specific heat data.

This empirical formula is a better fit to the data than the expected Schottky form for a 2DES in the absence of disorder [12]. As the electron temperatures involved are much less than the magnetoroton energy, the heat capacity due to these excitations will be exponentially small. It is probable therefore that we measure a heat capacity at $v=1 / 3$ that is due other degrees of freedom such as localised states and sample inhomogeneity.

Through the use of the Sommerfeld expansion [13] for the total energy of the 2DES and using the empirical relation for the heat capacity, we can estimate the form of the composite fermion density of states as a function of energy at $v=$ 1/3 (inset Fig. 5).

Knowing the temperature dependence of the specific heat of the 2DES we can now evaluate the energy absorbed from the ballistic phonons as a function of the temperature of the non-equilibrium phonons. We regard this absorbed energy as an indirect consequence of the excitation of the 2DES across the magnetoroton gap. The fast $(<20$ ns) decay of the excitations inside the

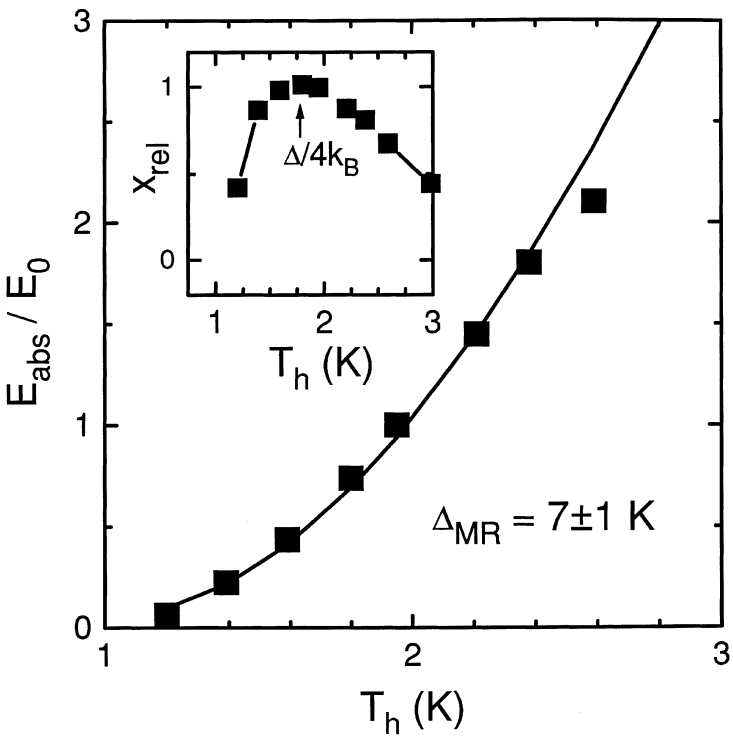

Fig. 6. Dependence of the energy absorbed by the 2DES at $v=1 / 3$ (Sample A) on the heater temperature $T_{\mathrm{h}}$. The solid line shows the fitted behaviour for excitation across a magnetoroton gap, $\Delta_{\mathrm{MR}}$. In the inset, the total phonon energy absorbed relative to the phonon energy emitted is shown as a function of $T_{\mathrm{h}}$.

2DES, i.e. without emission of high-energy phonons, eventually heats the 2DES to a temperature $T_{1}$. The total energy absorbed, $E_{\text {abs }}\left(T_{\mathrm{h}}\right)$, arbitrarily normalised to its value $E_{0}$ for $T_{\mathrm{h}}=1.95 \mathrm{~K}$ and $\tau=100 \mathrm{~ns}$, increases as $\left(\exp \left(-\Delta_{\mathrm{MR}} / k_{\mathrm{B}} T_{\mathrm{e}}\right)-1\right)^{-1}$ (Fig. 6). We find a magnetoroton energy gap of $7 \pm 1 \mathrm{~K}$, twice as high as that determined from transport experiments on the same sample. The energy gap measured by transport experiments is expected to be the larger energy gap at infinite wavevector rather than that at the magnetoroton minimum. Experimentally, it has been found that this measure of the energy gap is reduced by the presence of disorder. The phonon absorption results suggest that the energy required to create a magnetoroton from the strongly correlated Laughlin liquid is largely independent of the disorder in the sample. As the characteristic length scale involved in this process is the magnetic length it might be expected that the process is only sensitive to disorder that varies the properties of the 2DES on length scales shorter than this. 
Another method of showing the existence of the energy gap is to plot the ratio, $x_{\text {rel }}$, of the energy absorbed by the 2DES to the energy in the phonon pulse $E_{\mathrm{abs}} / E_{\mathrm{ph}}$ as a function of heater temperature (inset Fig. 6). For deformation potential coupling it would be expected that this curve peaks at $T_{\mathrm{p}} \approx \Delta_{\mathrm{MR}} / 4 k_{\mathrm{B}} T_{\mathrm{h}}$ (suggesting $\Delta_{\mathrm{MR}}=7.2 \pm 0.5 \mathrm{~K}$ ) where the dominant phonon energy in the blackbody spectrum coincides with the magnetoroton gap. The two experimentally determined values for the magnetoroton energy at $v=1 / 3$ are found to be the same to within experimental error.

For an infinitely thin 2DES, Girvin et al. [5] predict that the magnetoroton minimum at $v=$ $1 / 3$ will be at an energy of $c_{1 / 3} e^{2} / 4 \pi \varepsilon_{0} \varepsilon l_{\mathrm{B}}$ where $c_{1 / 3}=0.075$. Having determined $a_{0}$ and knowing the magnetic length at $v=1 / 3$ in sample A, the prediction for $c_{1 / 3}$ can be corrected for the effects of finite thickness [14]. The predicted value becomes $c_{1 / 3} \approx 0.042 \pm 0.002$. Expressing the experimental value in the reduced units, we determine that $c_{1 / 3}=0.036 \pm 0.006$. The agreement between the theoretical and experimental values supports the hypothesis that the ballistic phonon measurements are probing the magnetoroton gap at finite inplane wavevector.

\section{Conclusion}

In conclusion, we have performed the first ballistic angle-resolved phonon absorption experiments in the fractional quantum Hall and composite fermion regime. These are the first experiments, in this regime, in which a probe with a well-defined, finite, wavevector has been observed to couple to these strongly correlated electron liquids. Thus the technique provides an important new spectroscopic tool for the investigation of these novel states of matter.

\section{Acknowledgements}

We gratefully acknowledge funding for this research from the EPSRC and useful discussions with Dr. D. Lehmann and Dr. W. Dietsche.

\section{References}

[1] C.J. Mellor et al., Phys. Rev. Lett. 74 (1995) 2339.

[2] W. Kang et al., Phys. Rev. Lett. 75 (1995) 4106.

[3] B. Tieke et al., Phys. Rev. Lett. 76 (1995) 3640.

[4] E. Chow et al., Phys. Rev. Lett. 77 (1995) 1143.

[5] B.I. Halperin, P.A. Lee, N. Read, Phys. Rev. B 47 (1993) 7312.

[6] S.M. Girvin, A.H. MacDonald, P.M. Platzman, Phys. Rev. B 33 (1996) 1381.

[7] A. Pinczuk, B.S. Dennis, L.N. Pfeiffer, K. West, Phys. Rev. Lett. 70 (1993) 3983; H.D.M. Davies, J.C. Harris, J.F. Ryan, A.J.Turberfield, Phys. Rev. Lett. 78 (1997) 4095.

[8] U. Zeitler et al., Physica B (1998) to be published; J.E. Digby, Ph.D. Thesis, University of Nottingham, 1997.

[9] F. Rosch, O. Weis, Z. Phys. B 27 (1986) 33.

[10] A.J. Kent, R.E. Strickland, K.R. Strickland, M. Henini, Phys. Rev. B 54 (1996) 2019.

[11] Y.B. Kim, P.A. Lee, Phys. Rev. B 54 (1996) 2715.

[12] T. Chakraborty, P. Pietilainen, Phys. Rev. B 55 (1997) 1954.

[13] N.W. Ashcroft, N.D. Mermin, Solid State Physics, Appendix C, Saunders College Publishing, 1976.

[14] F.C. Zhang, S. Das Sarma, Phys. Rev. B 33 (1986) 2903. 